\title{
Fair allocation in evolving networks
}

Citation for published version (APA):

Navarro, N. (2003). Fair allocation in evolving networks. METEOR, Maastricht University School of Business and Economics. METEOR Research Memorandum No. 010 https://doi.org/10.26481/umamet.2003010

Document status and date:

Published: 01/01/2003

DOI:

10.26481/umamet.2003010

Document Version:

Publisher's PDF, also known as Version of record

\section{Please check the document version of this publication:}

- A submitted manuscript is the version of the article upon submission and before peer-review. There can be important differences between the submitted version and the official published version of record.

People interested in the research are advised to contact the author for the final version of the publication, or visit the DOI to the publisher's website.

- The final author version and the galley proof are versions of the publication after peer review.

- The final published version features the final layout of the paper including the volume, issue and page numbers.

Link to publication

\footnotetext{
General rights rights.

- You may freely distribute the URL identifying the publication in the public portal. please follow below link for the End User Agreement:

www.umlib.nl/taverne-license

Take down policy

If you believe that this document breaches copyright please contact us at:

repository@maastrichtuniversity.nl

providing details and we will investigate your claim.
}

Copyright and moral rights for the publications made accessible in the public portal are retained by the authors and/or other copyright owners and it is a condition of accessing publications that users recognise and abide by the legal requirements associated with these

- Users may download and print one copy of any publication from the public portal for the purpose of private study or research.

- You may not further distribute the material or use it for any profit-making activity or commercial gain

If the publication is distributed under the terms of Article $25 \mathrm{fa}$ of the Dutch Copyright Act, indicated by the "Taverne" license above, 


\title{
Fair Allocation in Evolving Networks
}

\author{
Noemí Navarro* \\ Universidad Carlos III de Madrid
}

February 2003

\begin{abstract}
We consider networks evolving over time within an infinite-horizon dynamic setting. Transitions from one network to another are given by a stationary transition probability matrix. We study the problem of fairly and efficiently allocating the value of a network at any point in time among its participants, assuming that agents discount the future by some common discount factor $\delta$. An allocation rule is called component efficient if it distributes the total value of a connected network among its participants and it is called expected fair if for every direct connection both participants expect to loose or gain the same amount in the future from breaking this connection at time zero. Our main result is that for every transition probability matrix and for almost every $\delta$ there exists a unique allocation rule which is component efficient and expected fair. We provide a formula to compute this allocation rule. In general, this allocation rule is different from a stage-wise application of the Myerson value. We also provide a sufficient condition on the transition probability matrix such that the component efficient and expected fair allocation rule is equal to the Myerson value.

Keywords: Myerson value, allocation rules, networks, Markov chain.

JEL Classification: C71, C79.
\end{abstract}

Noemí Navarro: Universidad Carlos III de Madrid, Departamento de Economía, Calle Madrid, 126, 28903 Getafe (Madrid), Spain. E-mail: snavarro@eco.uc3m.es. Fax: +34 91624 9875 .

${ }^{*}$ I wish to thank my supervisor, Andrés Perea, for his encouragement and very helpful comments. I thank Marco Slikker, Jordi Massó and Luis Corchón for their suggestions. Most of this work was written while the author was visiting the CRDE-CIREQ, Universite de Montreal, and the Department of Quantitative Economics at the University of Maastricht. I am grateful for their hospitality. Financial support from research grants FP1999 44428130 and BEC 2002-02194 from the Spanish Ministry of Science and Technology are gratefully acknowledged. The usual disclaimer applies. 


\section{Introduction}

We study situations in which network structures play an important role in the outcome of some economic activity. For example, networks may determine how information about job openings is spread among a population, they can represent a series of bilateral and binding contracts, or they can be a mechanism for exchanging goods or services which are not traded in markets.

Consider a network consisting of several connected sub-networks, to which we refer as connected components. Every connected component within a network has a value, which may be interpreted as the total utility obtained by the participants in this component. The standard model assumes that there are no externalities among connected components in a network. In other words, the total value which can be distributed among the participants of a connected component is independent of the network structure outside this component. In this work, we allow the value of a component to depend on the network structure outside. For instance, assume that agents represent firms in an industry. A network in this situation may consist of a series of bilateral contracts between firms. A group of firms in a connected component can be seen as a cartel. The value of a connected component is the total benefit achieved by the firms participating in this component and this value will depend on the network structure outside this component. Another example may be the case of job contact networks. Assume that job information is spread along a network, and all connected components have a positive probability of being informed about a vacancy in the economy. Therefore, if the network outside a component is very connected, there is a high probability that many people outside the component will hear about the vacancy. Thus, there will be more candidates for an open position and therefore the probability that someone in the connected component gets a job is lower. This means that the value of the connected component depends on the structure of the network outside ${ }^{1}$.

In this paper we assume that networks evolve over time within an infinite-horizon setting. For instance, in the industry example above, firms may sign new contracts or dissolve existing ones. At time zero there is an exogenously given network, and agents get their payoff from participating in this network according to an allocation rule which is time-independent. Afterwards, the transition to another network occurs according to a stationary transition probability matrix. At the new network, all agents receive a payoff according to the previously mentioned allocation rule. Afterwards, a transition to another network occurs, and so on. We assume that agents discount the future by some common discount factor $\delta$.

An allocation rule is said to be component efficient if it distributes the total value of a connected network among its participants and it is said to be expected fair if for every direct connection in the network both participants expect to loose or gain the same amount in the future from breaking this connection at time zero. This concept may be interpreted as an equalgains or equal bargaining power principle in which agents take the future into account. In our main result we show that for every transition probability matrix and for almost every discount factor $^{2}$ there exists a unique allocation rule which is component efficient and expected fair. As a preparatory step for this result we prove a lemma which may have interest in itself, namely that within a static context of networks with externalities there is always a unique allocation

\footnotetext{
${ }^{1}$ Calvo-Amengol and Jackson [4] analyse this situation, although using a different model than ours.

2 "For almost every discount factor" means that the result holds for all but finitely many $\delta$.
} 
rule which is component efficient and fair, thereby extending an earlier result by Myerson [9], Feldman [5] and Jackson and Wolinsky [7]. This rule is referred to as the Myerson value.

Myerson [9] has defined fairness in a situation where the surplus from cooperation is given by a TU game. An allocation rule is said to be fair ${ }^{3}$ if for every direct connection in the network, both participants gain or loose the same amount from breaking this connection. In this model, networks connecting the same group of agents have the same value. Jackson and Wolinsky [7] extended this model to a situation where different networks connecting the same group of agents may take different values. Feldman [5] extended the model defined by Myerson [9] to situations where the surplus from cooperation is given by a game in partition function form. Such games are an extension of TU games, where the value of a coalition may depend on the coalition structure outside ${ }^{4}$. Neither the model by Jackson and Wolinsky [7] is a special case of the model by Feldman [5], nor the opposite. Nevertheless, both papers have shown, in their respective settings, that there is a unique allocation rule which is component efficient and fair, extending the earlier result by Myerson [9].

We finally analyze two special classes of transition probability matrices. The first class, the class of "component dissolvable" transition probability matrices, enables us to provide a simpler formula than in the general case. In the second class, containing transition probability matrices satisfying the "sum-property", the unique component efficient and expected fair allocation rule is equal to the stage-wise application of the Myerson value.

At this stage we would like to discuss some related literature, mainly dealing with probabilistic networks. Calvo et al. [3] extend the model of Myerson [9] to a situation where each pair of players can directly communicate with some positive probability. These probabilities are assumed to be independent. The probability that a certain graph is realized is thus binomial, although different direct connections may have different probabilities. Their main result is the following: the expected payoff to every player is component efficient ${ }^{5}$ and fair if and only if the allocation rule at each realized graph coincides with the Myerson value.

Bala and Goyal [1] study the problem of strategic network formation. In their model the allocation rule is fixed and there is a constant probability that a direct connection fails to transmit correct information. The models presented by Calvo et al. [3] and by Bala and Goyal [1] satisfy the "sum-property" mentioned above.

Jackson and Watts [6] analyze the stochastic evolution of networks. In their work, the allocation rule is fixed and the transition probabilities depend on that allocation rule. At any point in time, a pair of players is chosen according to some predetermined probability distribution. If they are directly connected in the actual graph, they can break this connection and if they are not, they can choose to create it. Players are myopic and consider only the improvement in their stage payoff at the new network relative to the current one. After a decision is taken, there is a small probability of trembling, or making mistakes.

The model in our paper could be extended to a situation where the transition probabilities are related to the allocation rule. It may be reasonable to assume that the probability of adding

\footnotetext{
${ }^{3}$ Jackson and Wolinsky [7] call this property Equal Bargaining Power.

${ }^{4}$ The definition of a game in partition function form is due to Lucas and Thrall [8], and several allocation rules have been defined in this context (see, for example, Myerson [10], Bolger [2] and Feldman [5]).

${ }^{5} \mathrm{With}$ respect to the expected value of a component.
} 
(resp. deleting) a new (resp. present) direct connection to the existing graph is increasing in the discounted expected gains from creating (resp. deleting) that link by the two players involved in it. Such analysis is beyond the scope of this paper, although it may be an interesting application for network formation problems.

This paper is organized as follows. In Section 2 the static model is presented, whereas Section 3 deals with the dynamic model. Section 4 presents the main result. In Section 5 we present some special cases of transition probability matrices and finally we provide an example in Section 6.

\section{Static Network Environments}

Let $N=\{1, \ldots, n\}$ be a finite set of players. A subset $S \subseteq N$ is called a coalition. Let $2^{N}$ be the set of all possible coalitions in $N$. We will assume that there are network relations among players in $N$, formally represented by an undirected graph. Here, an undirected graph $g$ is a set of unordered pairs $(i, j)$, where $i, j \in N$, and $i \neq j$. Throughout the paper, each unordered pair $(i, j)$ will be referred to as a link.

Let $g^{N}$ be the set of all unordered pairs in $N$, that is, the full graph over $N$. Let $g \cup(i, j)$ denote the graph resulting from adding the link $(i, j)$ to the existing graph $g$, while $g \backslash(i, j)$ denotes the graph resulting from deleting the link $(i, j)$ from the graph $g$. We define the restriction of $g$ to a coalition $S$ as $g \mid S=\{(i, j) \in g: i \in S$ and $j \in S\}$. Note that $g \mid S \subseteq g$ and $g \mid N=g$. A coalition $T \subseteq S$ is called a connected component of $S$ in $g$ if: (1) for every two players in $T$, there is a path, that is, a set of consecutive links, in $g \mid S$ connecting them, and (2) for any player $i$ in $T$ and any player $j$ not in $T$, there is no path in $g \mid S$ which connects them. Let $S \mid g$ be the set of connected components of $S$ in $g$. Note that $S \mid g$ is a partition on $S$. Similarly, we may define $N \mid g$. Let $G$ be the set of all possible graphs on $N$.

Now assume that for every graph $g$ and every connected component $S \in N \mid g$, there is a value $w(S, g)$ which can be perfectly distributed among the agents in $S$. A function $w$, which to every graph $g$ and every component $S$ in $N \mid g$ assigns a value $w(S, g)$, is called a value function. The value which can be distributed among the players in $N$ when the graph is $g$ is thus given by $\sum_{S \in N \mid g} w(S, g)$. Notice that we allow for externalities between the connected components, for $w(S, g)$ may depend on the structure of $g$ outside $S$. As we have said in the introduction, this is different from the standard assumption. Myerson [9] restricts attention to value functions for which $w(S, g)$ does not depend on $g$. Jackson and Wolinsky [7] relaxed the previous restriction by imposing that the value $w(S, g)$ only depends on the network structure inside $S$. Formally, $w(S, g)=w\left(S, g^{\prime}\right)$ for every pair of graphs $g$ and $g^{\prime}$ with $g\left|S=g^{\prime}\right| S$. Feldman [5] extends the model by Myerson [9] by assuming that the value $w(S, g)$ only depends on the set of connected components induced by $g$. That is, $w(S, g)=w\left(S, g^{\prime}\right)$ if $N|g=N| g^{\prime}$. Neither Jackson and Wolinsky [7] is a special case of Feldman [5] nor the opposite. By imposing no restrictions on the value function, we assume that the value of a connected component $S$ may vary if the network structure inside varies, if the set of connected components $N \mid g$ varies, or if the network structure outside varies. 
From now on, the value function is assumed to be fixed. An allocation rule is a function $y$ which assigns to every graph $g \in G$ some payoff vector $y(g) \in \Re^{n}$.

Definition 2.1. An allocation rule $y$ is called component efficient if for every graph $g \in G$ and every connected component $S \in N \mid g$

$$
\sum_{i \in S} y_{i}(g)=w(S, g)
$$

Definition 2.2. An allocation rule $y$ is called fair if for every graph $g \in G$ and every link $(i, j) \in g$

$$
y_{i}(g)-y_{i}(g \backslash(i, j))=y_{j}(g)-y_{j}(g \backslash(i, j)) .
$$

By fairness we impose that for each link in a graph, both participants should gain or loose the same amount from breaking this link.

We now present an extension of a result obtained by Myerson [9], Feldman [5] and Jackson and Wolinsky [7] to the general case.

Theorem 2.3. For every value function $w$ there is a unique component efficient and fair allocation rule.

Proof of Theorem 2.3. Let $y$ be an allocation rule. For the sequel, the allocation rule $y$ is considered to be a vector in $\Re^{N \times G}$. It is easily seen that if $y$ is component efficient and fair, then its elements have to satisfy a system of linear equations. Consider only the equations corresponding to fairness and let $y_{i, g}$ be the payoff received by player $i$ in graph $g$. Then, for every graph $g \in G$ and for every link $(i, j) \in g$ we have the following fairness equation:

$$
y_{i, g}-y_{i, g \backslash(i, j)}-y_{j, g}+y_{j, g \backslash(i, j)}=0 .
$$

Let a cycle $C \subseteq g$ be a path in $g$ such that: (1) the number of links in the path is at least three and (2) the first and last player are the same. A cycle $C \in g$ is a minimal cycle in $g$ if no subset of $C$ forms a cycle in $g$. We show now that for every graph $g$ and every minimal cycle $C$ in $g$, the fairness equations corresponding to the links in $C$ are linearly dependent.

Let $C$ be a minimal cycle in $g$. For any link $(i, j) \in C, C \backslash(i, j)$ is a path in $g$ going from player $i$ to player $j$. Thus, $C \backslash(i, j)=\left\{\left(i_{1}, i_{2}\right),\left(i_{2}, i_{3}\right), \ldots,\left(i_{K-1}, i_{K}\right)\right\}$, with $i_{1}=i$ and $i_{K}=j$. Note that every player $i_{k}$ in $C$ is involved in exactly two links. Let $l^{+}\left(i_{k}\right)$ be $\left(i_{k}, i_{k+1}\right)$ for all $k \neq K$, let $l^{-}\left(i_{k}\right)=\left(i_{k-1}, i_{k}\right)$ for all $k \neq 1$ and let $l^{+}\left(i_{K}\right)=l^{-}\left(i_{1}\right)=\left(i_{K}, i_{1}\right)$. For every $l \in C$ and $\tilde{g} \subseteq g$, with $l \in \tilde{g}$, define the row vector $f(\tilde{g}, l) \in \Re^{N \times G}$ as $f(\tilde{g}, l)=\left[f_{i, g^{\prime}}(\tilde{g}, l)\right]_{i \in N, g^{\prime} \in G}$, where

$$
f_{i, g^{\prime}}(\tilde{g}, l)=\left\{\begin{array}{cl}
1, & \text { if }\left[l=l^{+}(i) \text { and } \tilde{g}=g^{\prime}\right] \text { or }\left[l=l^{-}(i) \text { and } \tilde{g}=g^{\prime} \cup l\right] \\
-1, & \text { if }\left[l=l^{+}(i) \text { and } \tilde{g}=g^{\prime} \cup l\right] \text { or }\left[l=l^{-}(i) \text { and } \tilde{g}=g^{\prime}\right] \\
0, & \text { otherwise. }
\end{array}\right.
$$


Note that the fairness equation corresponding to graph $\tilde{g}$ and link $l \in \tilde{g}$ can be written as

$$
f(\tilde{g}, l) \times y=0 .
$$

We now prove that

$$
\sum_{l \in C} \sum_{\substack{\tilde{g} \subseteq g \\ l \in \tilde{g}}}(-1)^{|\tilde{g}|} f(\tilde{g}, l)=0 .
$$

We therefore must show that

$$
\sum_{l \in C} \sum_{\substack{\tilde{g} \subseteq g \\ l \in \tilde{g}}}(-1)^{|\tilde{g}|} f_{i, g^{\prime}}(\tilde{g}, l)=0
$$

for every player $i$ and every graph $g^{\prime} \in G$. If player $i$ is not in $C$ or if graph $g^{\prime}$ is not a subgraph of $g$, then, $f_{i, g^{\prime}}(\tilde{g}, l)=0$ for every graph $\tilde{g} \subseteq g$ and every link $l$ in $C$. Therefore, the sum in (2.1) above is zero. The only relevant cases are thus when $i$ is a player in the minimal cycle $C$ and when $g^{\prime}$ is a subgraph of $g$.

Fix a player $i$ in $C$ and a graph $g^{\prime} \subseteq g$. We distinguish four cases.

(1) Assume $l^{+}(i)$ and $l^{-}(i) \in g^{\prime}$. Then, for every link $l \in C$ and every graph $\tilde{g} \subseteq g$ with $l \in \tilde{g}$ we have

$$
f_{i, g^{\prime}}(\tilde{g}, l)=\left\{\begin{array}{cl}
1, & \text { if } l=l^{+}(i) \text { and } \tilde{g}=g^{\prime} \\
-1, & \text { if } l=l^{-}(i) \text { and } \tilde{g}=g^{\prime} \\
0, & \text { otherwise. }
\end{array}\right.
$$

Therefore,

$$
\sum_{l \in C} \sum_{\substack{\tilde{g} \subseteq g \\ l \in \tilde{g}}}(-1)^{|\tilde{g}|} f_{i, g^{\prime}}(\tilde{g}, l)=(-1)^{\left|g^{\prime}\right|}-(-1)^{\left|g^{\prime}\right|}=0 .
$$

(2) Assume $l^{+}(i) \in g^{\prime}$ and $l^{-}(i) \notin g^{\prime}$. Then, for every link $l \in C$ and every graph $\tilde{g} \subseteq g$ with $l \in \tilde{g}$ we get

$$
f_{i, g^{\prime}}(\tilde{g}, l)= \begin{cases}1, & \text { if }\left[l=l^{+}(i) \text { and } \tilde{g}=g^{\prime}\right] \text { or }\left[l=l^{-}(i) \text { and } \tilde{g}=g^{\prime} \cup l^{-}(i)\right] \\ 0, & \text { otherwise. }\end{cases}
$$

Thus,

$$
\sum_{l \in C} \sum_{\substack{\tilde{g} \subseteq g \\ l \in \tilde{g}}}(-1)^{|\tilde{g}|} f_{i, g^{\prime}}(\tilde{g}, l)=(-1)^{\left|g^{\prime}\right|}+(-1)^{\left|g^{\prime}\right|+1}=0 .
$$

(3) Assume $l^{+}(i) \notin g^{\prime}$ and $l^{-}(i) \in g^{\prime}$. Then, for every link $l \in C$ and every graph $\tilde{g} \subseteq g$ with $l \in \tilde{g}$ we obtain

$$
f_{i, g^{\prime}}(\tilde{g}, l)=\left\{\begin{array}{cl}
-1, & \text { if }\left[l=l^{-}(i) \text { and } \tilde{g}=g^{\prime}\right] \text { or }\left[l=l^{+}(i) \text { and } \tilde{g}=g^{\prime} \cup l^{+}(i)\right] \\
0, & \text { otherwise. }
\end{array}\right.
$$


Again,

$$
\sum_{l \in C} \sum_{\substack{\tilde{g} \subseteq g \\ l \in \tilde{g}}}(-1)^{|\tilde{g}|} f_{i, g^{\prime}}(\tilde{g}, l)=-(-1)^{\left|g^{\prime}\right|}-(-1)^{\left|g^{\prime}\right|+1}=0 .
$$

(4) Assume $l^{+}(i)$ and $l^{-}(i) \notin g^{\prime}$. Then, for every link $l \in C$ and every graph $\tilde{g} \subseteq g$ with $l \in \tilde{g}$ it holds that

$$
f_{i, g^{\prime}}(\tilde{g}, l)=\left\{\begin{array}{cl}
-1, & \text { if } l=l^{+}(i) \text { and } \tilde{g}=g^{\prime} \cup l^{+}(i) \\
1, & \text { if } l=l^{-}(i) \text { and } \tilde{g}=g^{\prime} \cup l^{-}(i) \\
0, & \text { otherwise. }
\end{array}\right.
$$

Hence,

$$
\sum_{l \in C} \sum_{\substack{\tilde{g} \subseteq g \\ l \in \tilde{g}}}(-1)^{|\tilde{g}|} f_{i, g^{\prime}}(\tilde{g}, l)=-(-1)^{\left|g^{\prime}\right|+1}+(-1)^{\left|g^{\prime}\right|+1}=0 .
$$

But this means that for any link $l$ in $C$, the fairness equation corresponding to link $l$ and graph $g$ is a linear combination of the fairness equations corresponding to all pairs $\left(\tilde{g}, l^{\prime}\right)$ with $\tilde{g} \subseteq g, l^{\prime} \in C$ and $l^{\prime} \in \tilde{g}$.

Therefore, for any graph $g$ with cycles we can do the following. First choose a minimal cycle in $g$. As we have shown above, one equation corresponding to one link in this minimal cycle can be deleted, say $f\left(g, l_{1}\right)$. Consider now the resulting system in which $f\left(g, l_{1}\right)$ has been deleted. If $g \backslash l_{1}$ has cycles, we choose another minimal cycle in $g \backslash l_{1}$ and delete the equation corresponding to one of the links in it, and so on. After a finite number of steps, the equations implied by fairness in graph $g$ which are left correspond to links in $g$ which form a spanning forest of $g$.

By component efficiency we obtain the equation

$$
\sum_{i \in S} y_{i}(g)=w(S, g)
$$

for any graph $g$ and any connected component $S \in N \mid g$.

For every graph $g$ in $G$ select a spanning forest ${ }^{6}$ of $g$, say $F(g)$. Define the system of equations $S_{f}$ as a system which contains all equations $f(g, l)$ such that $l \in F(g)$ for every $g$ in $G$. It can be checked that all equations in $S_{f}$ are linearly independent, and we have seen that any fairness equation which is not in $S_{f}$ can be written as a linear combination of the equations in $S_{f}$. It is easily seen that the equations in $S_{f}$ together with the component efficiency equations are linearly independent, with the same number of equations as unknowns. Hence, the system of equations defined by component efficiency and fairness has a unique solution. This completes the proof of Theorem 2.3.

Throughout the paper, this component efficient and fair allocation rule will be referred to as the Myerson value and it will be denoted by $m$.

\footnotetext{
${ }^{6}$ If $g$ has no cycles, the unique spanning forest of $g$ is trivially itself.
} 
We now provide an explicit formula for the Myerson value. In order to do so, we need the following definitions. Let $\Pi(N)$ be the set of partitions of $N$. Thus, $P=\left\{S_{1}, \ldots, S_{k}\right\} \in \Pi(N)$ if (1) $S_{r} \neq \varnothing$ for all $r=1, \ldots, k,(2) \bigcup_{r=1}^{k} S_{r}=N$ and (3) $S_{r} \cap S_{t}=\varnothing$ if $r \neq t$. An embedded coalition is a pair $(S, P)$ where $S \in P \in \Pi(N)$. Let $E(N)$ be the set of all possible embedded coalitions in $N$. Let $|S|$ be the number of players in $S$ and $|P|$ the number of non-empty coalitions in $P$.

A game in partition function form (PFF) is a pair $\langle N, v\rangle$ where $v$ is a function which assigns to every $(S, P) \in E(N)$ a real number $v(S, P)$. Given the coalitional structure $P$, the real number $v(S, P)$ is the value which can be perfectly transferred across players in $S$ when the coalitional structure is given by $P$. The function $v$ is called a coalitional value function.

An allocation rule in this context is a function $\varphi$ which assigns to every game in $\operatorname{PFF}\langle N, v\rangle$ a payoff vector $\varphi(N, v) \in \Re^{n}$.

Definition 2.4. The Myerson value for games in PFF is the allocation rule which to every game in PFF $\langle N, v\rangle$ assigns the payoff vector $\varphi(N, v)$ given by

$$
\varphi_{i}(N, v)=\sum_{(S, P) \in E(N)}(-1)^{|P|-1}(|P|-1) !\left(\frac{1}{n}-\sum_{\substack{\tilde{S} \in P \\ \tilde{S} \neq S \\ i \notin \tilde{S}}} \frac{1}{(|P|-1)(n-|\tilde{S}|)}\right) v(S, P),
$$

for all players $i$.

For the sequel, the Myerson value for games in $\mathrm{PFF}$ will be denoted by $\varphi^{M}$.

Now we proceed to apply the definitions mentioned above to the context of static networks with externalities. Given a value function $w$ and a graph $g$ let the auxiliary game in $\operatorname{PFF}\left\langle N, U_{g}\right\rangle$ be given by

$$
U_{g}(S, P)=\sum_{R \in S \mid g} w(R, g \mid P)
$$

where $g\left|P=\bigcup_{T \in P} g\right| T$.

We now present an explicit formula for the Myerson value in the general case.

Theorem 2.5. The Myerson value is the allocation rule which assigns to every graph $g$ the allocation $\varphi^{M}\left(N, U_{g}\right)$.

The proof of this theorem is given in the appendix.

\footnotetext{
${ }^{7}$ This allocation rule was defined and axiomatically characterized by Myerson [10]. It is an extension of the Shapley value to games in PFF.
} 


\section{Dynamic Network Environments}

Now assume that we have an infinite number of stages, and at each stage $t$ there is a transition probability from the existing graph $g_{t}$ to any other graph $g_{t+1}$ in the following stage. If we assume that these probabilities do not depend on the period $t$, the graph at each stage follows an infinite stationary Markov chain with transition probabilities given by the matrix

$$
P=\left[P\left(g_{1} \mid g_{2}\right)\right]_{g_{1}, g_{2} \in G},
$$

where $P\left(g_{1} \mid g_{2}\right)$ is the probability of arriving at $g_{1}$ conditional on $g_{2}$ being the actual graph.

Assume that players receive some payoff at each stage according to some fixed allocation rule $y$. Suppose that players discount the future by a factor $0<\delta<1$, and define

$$
P^{\delta, \infty}=\sum_{t=0}^{\infty} \delta^{t} P^{t} .
$$

Here, $P^{\delta, \infty}\left(g_{1} \mid g_{2}\right)$ may be interpreted as the limit-discounted probability of reaching graph $g_{1}$ when starting with graph $g_{2}$.

For any allocation rule $y$, and any initial graph $g$, the discounted expected payoff for player $i$ is given by

$$
x_{i}(y, g, \delta, P)=\sum_{g^{\prime}} P^{\delta, \infty}\left(g^{\prime} \mid g\right) y_{i}\left(g^{\prime}\right)
$$

As is well known, the matrix $(I-\delta P)$ has an inverse, namely $(I-\delta P)^{-1}=P^{\delta, \infty}$. Here, $I$ is the identity matrix. Let $x(y, g, \delta, P)=\left[x_{i}(y, g, \delta, P)\right]_{i \in N}$. From now on, we simply write $x(y, g)$ instead of $x(y, g, \delta, P)$, since $P$ and $\delta$ are always fixed.

Definition 3.1. An allocation rule $y$ is called expected fair if for every $g \in G$ and every link $(i, j) \in g$

$$
x_{i}(y, g)-x_{i}(y, g \backslash(i, j))=x_{j}(y, g)-x_{j}(y, g \backslash(i, j)) .
$$

By expected fairness we restrict attention to allocation rules where for every link in a graph, both players expect to gain or loose the same from breaking such a connection at time zero. It is thus a forward-looking extension of the fairness property to situations where networks evolve over time.

\section{The Main Result}

We show now that for all transition probability matrices $P$ and for almost every $\delta$, there exists a unique allocation rule which is component efficient and expected fair. In order to state the result we need the following definitions.

Let $x$ be the expected payoff vector induced by an allocation rule $y$. That is, $x$ assigns to every player $i$ and every graph $g$ some expected payoff $x_{i, g}$. Let $(P \circ x)(i, g)=\sum_{g^{\prime}} P\left(g^{\prime} \mid g\right) x_{i, g^{\prime}}$ 
be the expected payoff player $i$ gets in the next period if the actual graph is $g$ and the payoffs are given by $x$.

Let $\left(W_{P} \circ x\right)(S, g)=\sum_{i \in S}(P \circ x)(i, g)$, for every $S \in N \mid g$. Note that the operator $P$ transforms an allocation rule $x$ into another allocation rule $P \circ x$, and that $W_{P}$ transforms an allocation $x$ into a value function $W_{P} \circ x$. Since $\left(W_{P} \circ x\right)$ is a value function, we can compute its Myerson value. Let $\left(m \circ W_{P}\right) \circ x$ be the Myerson value applied to $W_{P} \circ x$. It is easily seen that $\left(W_{P} \circ x\right)$ is linear in $x$. Furthermore, the function $m$ is linear in the value function. Hence, $\left(m \circ W_{P}\right) \circ x$ is linear in $x$. Note that $\left(m \circ W_{P}\right)$ is an operator which transforms an allocation rule $x$ into another allocation rule $\left(m \circ W_{P}\right) \circ x$.

Let $I$ be the identity operator. Since $P$ and $\left(m \circ W_{P}\right)$ transform allocation rules into allocation rules, so do the operators $(I-\delta P)$ and $\left(I-\delta\left(m \circ W_{P}\right)\right)^{-1}$, if the latter exists.

Theorem 4.1. (1) The operator $\left(I-\delta\left(m \circ W_{P}\right)\right)$ has an inverse for almost all $\delta$, and (2) for all these $\delta$ we have that there is a unique allocation rule $f$ which is component efficient and expected fair, and it is given by

$$
f(g)=\left((I-\delta P) \circ\left(I-\delta\left(m \circ W_{P}\right)\right)^{-1} \circ m\right)(g),
$$

for all graphs $g$.

Proof of Theorem 4.1. We proceed to prove (1) first. Let $A$ be the matrix associated with the linear operator $\left(m \circ W_{P}\right)$. Then, $\left(I-\delta\left(m \circ W_{P}\right)\right)$ has an inverse if and only if $|I-\delta A| \neq 0$. We have that $|I-\delta A|=0$ if and only if $\frac{1}{\delta}$ is an eigenvalue of $A$. Therefore, the operator $\left(I-\delta\left(m \circ W_{P}\right)\right)$ has an inverse for every $\delta$ except for a (possibly empty) finite set of points. This completes the proof of (1).

(2) Assume that $\delta$ is such that the operator $\left(I-\delta\left(m \circ W_{P}\right)\right)$ has an inverse. Let $x^{*}$ be the allocation rule given by

$$
x^{*}(g)=\left(\left(I-\delta\left(m \circ W_{P}\right)\right)^{-1} \circ m\right)(g),
$$

for all $g$. We shall prove the following three steps. First, that the allocation rule $f$ in Theorem 4.1 generates $x^{*}$ as the expected payoff vector, i.e., $x^{*}(g)=x(f, g)$. Second, we prove that an allocation rule $y$ is component efficient with respect to $w$ and expected fair if and only if the induced discounted expected payoff vector $x(y)$ is the Myerson value of an auxiliary value function $W_{x(y)}$. Finally, we show that $x^{*}$ is the unique solution to $x=m\left(W_{x}\right)$, where $m\left(W_{x}\right)$ is the Myerson value applied to the value function $W_{x}$. For the sequel, whenever $m$ is written it is assumed to be $m(w)$, i. e., the Myerson value applied to the value function $w$.

Claim 1. The allocation rule

$$
f=(I-\delta P) \circ\left(I-\delta\left(m \circ W_{P}\right)\right)^{-1} \circ m
$$

generates $x^{*}$ as the expected payoff vector. 
Proof of Claim 1. By definition, $P^{\delta, \infty}=(I-\delta P)^{-1}=I+\delta P P^{\delta, \infty}$. Therefore, for any allocation rule $y$ and its induced discounted expected vector $x(y)$ we have

$$
x(y)=y+\delta P \circ x(y),
$$

which yields

$$
y=(I-\delta P) \circ x(y)
$$

Since

$$
f=(I-\delta P) \circ x^{*}
$$

it follows that $f$ generates $x^{*}$ as discounted expected payoff vector. This completes the proof of Claim 1.

Let $x$ be an allocation rule. Given $x$, we can define the following value function:

$$
W_{x}(S, g)=w(S, g)+\delta\left(W_{P} \circ x\right)(S, g),
$$

for all $g$ and for all $S \in N \mid g$. Let $m\left(W_{x}, g\right)$ be the Myerson value for $W_{x}$ at graph $g$.

Claim 2. The allocation rule $y$ is component efficient with respect to $w$ and expected fair if and only if $x(y)=m\left(W_{x(y)}\right)$.

Proof of Claim 2. We show first that if $y$ is component efficient and expected fair, then $x(y)=m\left(W_{x(y)}\right)$. Let $x=x(y)$. Since $P^{\delta, \infty}=I+\delta P P^{\delta, \infty}$,

$$
x_{i}(g)=y_{i}(g)+\delta \sum_{g^{\prime}} P\left(g^{\prime} \mid g\right) x_{i}\left(g^{\prime}\right)
$$

for all graphs $g$ and all players $i$.

If $y$ is component efficient with respect to $w$, then

$$
\sum_{i \in S} x_{i}(g)=w(S, g)+\delta \sum_{g^{\prime}} P\left(g^{\prime} \mid g\right) \sum_{i \in S} x_{i}\left(g^{\prime}\right)=W_{x}(S, g)
$$

for all $S \in N \mid g$. In other words, if $y$ is component efficient with respect to $w$, then $x$ is component efficient with respect to $W_{x}$.

On the other hand, if $y$ is expected fair, then $x$ is fair. But, there is only one allocation rule which is component efficient with respect to $W_{x}$ and fair, namely $m\left(W_{x}\right)$.

Now we prove that if $m\left(W_{x}, g\right)=x(g)$ for all graphs $g$, then the allocation rule $y$ which induces $x$ is component efficient and expected fair. If $m\left(W_{x}, g\right)=x(g)$ for all graphs $g$, then, for all graphs $g$ and all $S \in N \mid g$,

$$
\sum_{i \in S} x_{i}(g)=W_{x}(S, g)=w(S, g)+\delta \sum_{g^{\prime}} P\left(g^{\prime} \mid g\right) \sum_{i \in S} x_{i}\left(g^{\prime}\right)
$$


and

$$
\sum_{i \in S} y_{i}(g)=\sum_{i \in S} x_{i}(g)-\delta \sum_{g^{\prime}} P\left(g^{\prime} \mid g\right) \sum_{i \in S} x_{i}\left(g^{\prime}\right)=w(S, g)
$$

Therefore, the allocation rule $y$ which generates $x$ is component efficient. On the other hand, the allocation rule $y$ generating $x$ is also expected fair, for $x$ is fair. This completes the proof of Claim 2.

Claim 3. There is a unique allocation rule $x$ such that $x=m\left(W_{x}\right)$, namely

$$
x^{*}=\left(I-\delta\left(m \circ W_{P}\right)\right)^{-1} \circ m .
$$

Proof of Claim 3. By definition of $W_{x}$ above and by linearity of the Myerson value, $m\left(W_{x}\right)=$ $m+\delta\left(m \circ W_{P}\right) \circ x$. As we have seen before, $\left(m \circ W_{P}\right)$ is linear in $x$. Thus, if there is an $x$ such that $x=m\left(W_{x}\right)$, then it has to satisfy the linear equation

$$
\left(I-\delta\left(m \circ W_{P}\right)\right) \circ x=m .
$$

Note that if $\delta$ is such that $\left(I-\delta\left(m \circ W_{P}\right)\right)$ has an inverse, the solution to such equation is unique, namely $x^{*}=\left(I-\delta\left(m \circ W_{P}\right)\right)^{-1} \circ m$. This completes the proof of Claim 3 .

By Claims 2 and 3, the allocation rule which generates $x^{*}=\left(I-\delta\left(m \circ W_{P}\right)\right)^{-1} \circ m$ as discounted expected payoff vector is the unique allocation rule which is component efficient and expected fair. By Claim 1, the unique allocation rule which generates $x^{*}$ is $f$ and therefore it is the unique allocation rule which is component efficient and expected fair. This completes the proof of (2) and therefore it completes the proof of Theorem 4.1.

Note that Theorem 4.1 implies that there always exists a $\bar{\delta} \in(0,1)$ such that for every $\delta$ in $(\bar{\delta}, 1)$ there is a unique allocation rule which is component efficient and expected fair, namely the allocation rule $f$ given by

$$
f(g)=\left((I-\delta P) \circ\left(I-\delta\left(m \circ W_{P}\right)\right)^{-1} \circ m\right)(g),
$$

for all graphs $g$.

\section{Special Dynamic Processes}

We consider now two types of dynamic processes for which an easier formula to compute the unique component efficient and expected fair allocation rule can be provided.

For any graph $g \in G$, let the component closure of $g$, denoted by $c l(g)$, be $c l(g)=\bigcup_{S \in N \mid g} g^{S}$, where $g^{S}$ stands for the full graph over $S$.

Definition 5.1. The transition probability matrix $P$ is said to be component dissolving if for every pair of graphs $g^{\prime}, g \in G, P\left(g^{\prime} \mid g\right)>0$ only if $g^{\prime} \subseteq c l(g)$. 
Component dissolvability thus implies that the only new connections which are allowed are the ones between players who already belonged to the same component. Notice that with this assumption $P(\{\varnothing\} \mid\{\varnothing\})=1$.

A special case of component dissolvability is the case of dissolving networks, in which no new links can be built.

Let the value function $W$ be given by

$$
W(S, g)=\sum_{g^{\prime} \subseteq c l(g)} P^{\delta, \infty}\left(g^{\prime} \mid g\right) \sum_{R \in S \mid g^{\prime}} w\left(R, g^{\prime}\right),
$$

for every graph $g$ and every $S \in N \mid g$. Note that, since $S \in N \mid g$ and $g^{\prime} \subseteq \operatorname{cl}(g)$, every $R \subseteq S$ with $R \in S \mid g^{\prime}$ satisfies $R \in N \mid g^{\prime}$.

Theorem 5.2. Let the transition probability matrix $P$ be component dissolving. Then, for every $\delta$ there is a unique allocation rule $f$ which is component efficient and expected fair, namely

$$
f=(I-\delta P) \circ m(W) .
$$

Before stating the proof, we would like to mention the following. Note that this formula is easier than the one presented in Theorem 4.1, since computing the expression $\left(I-\delta\left(m \circ W_{P}\right)\right)^{-1} \circ m$ is a more complex task than computing $m(W)$. In order to compute $m(W)$, we need to find out $W$, which is the discounted expected value of $w$, and compute its Myerson value. On the other hand, in the general formula, we need to compute first the composed operator $m \circ W_{P}$ and, secondly, once $\left(I-\delta\left(m \circ W_{P}\right)\right)$ is obtained, we must take the inverse and apply it to $m$.

Proof of Theorem 5.2. We proceed to prove first that if the transition probability matrix is component dissolving and if an allocation rule $y$ is component efficient and expected fair, then $x(y)=m(W)$. Let $x=x(y)$. Then,

$$
x_{i}(g)=\sum_{g^{\prime}} P^{\delta, \infty}\left(g^{\prime} \mid g\right) y_{i}\left(g^{\prime}\right)
$$

for all graphs $g$ and all players $i$.

If $y$ is component efficient with respect to $w$, then

$$
\sum_{i \in S} x_{i}(g)=\sum_{g^{\prime}} P^{\delta, \infty}\left(g^{\prime} \mid g\right) \sum_{i \in S} y_{i}\left(g^{\prime}\right)=\sum_{g^{\prime} \subseteq c l(g)} P^{\delta, \infty}\left(g^{\prime} \mid g\right) \sum_{R \in S \mid g^{\prime}} w\left(R, g^{\prime}\right)=W(S, g),
$$

for all $S \in N \mid g$. In other words, if $y$ is component efficient with respect to $w$, then $x$ is component efficient with respect to $W$.

On the other hand, if $y$ is expected fair, then $x$ is fair. Since the unique allocation rule which is component efficient with respect to $W$ and fair is $m(W)$ it follows that $x=m(W)$.

Given that the allocation rule

$$
f=(I-\delta P) \circ m(W)
$$


is the unique allocation rule which generates $m(W)$ as expected discounted vector, it remains to prove that $f$ as defined above is indeed component efficient and fair. Since $P^{\delta, \infty}=I+\delta P P^{\delta, \infty}$,

$$
W(S, g)=w(S, g)+\delta \sum_{g^{\prime} \subseteq c l(g)} P\left(g^{\prime} \mid g\right) \sum_{R \in S \mid g^{\prime}} W\left(R, g^{\prime}\right)
$$

for all graphs $g$ and all $S \in N \mid g$.

By definition of the allocation rule $f$ and by component efficiency of $m(W)$,

$$
\begin{aligned}
\sum_{i \in S} f_{i}(g) & =\sum_{i \in S} m_{i}(W, g)-\delta \sum_{g^{\prime} \subseteq c l(g)} P\left(g^{\prime} \mid g\right) \sum_{i \in S} m_{i}\left(W, g^{\prime}\right)= \\
& =W(S, g)-\delta \sum_{g^{\prime} \subseteq c l(g)} P\left(g^{\prime} \mid g\right) \sum_{R \in S \mid g^{\prime}} W\left(R, g^{\prime}\right)=w(S, g) .
\end{aligned}
$$

Therefore, the allocation rule $f$ is indeed component efficient. On the other hand, the allocation rule $f$ generating $m(W)$ is also expected fair, for $m(W)$ is fair. This completes the proof of Theorem 5.2

We shall now provide a condition under which the unique component efficient and expected fair allocation rule is equal to the Myerson value.

Definition 5.3. The probability transition matrix $P$ is said to satisfy the sum-property if for every graph $g_{1}, g_{2} \in G$ and every link $(i, j) \notin\left(g_{1} \cup g_{2}\right)$

$$
P\left(g_{1} \mid g_{2}\right)+P\left(g_{1} \cup(i, j) \mid g_{2}\right)=P\left(g_{1} \mid g_{2} \cup(i, j)\right)+P\left(g_{1} \cup(i, j) \mid g_{2} \cup(i, j)\right) .
$$

Theorem 5.4. Let $P$ satisfy the sum-property. Then, for every $\delta$ there is a unique allocation rule $f$ which is component efficient and expected fair, namely $f(g)=m(g)$, for all graphs $g$.

Proof of Theorem 5.4. Let $P$ satisfy the sum-property. We will show that if $f$ is component efficient and expected fair, then it is the Myerson value of $w$. Let

$$
x_{i}(f, g)=\sum_{g^{\prime}} P^{\delta, \infty}\left(g^{\prime} \mid g\right) f_{i}\left(g^{\prime}\right)=f_{i}(g)+\delta \sum_{g^{\prime}} P\left(g^{\prime} \mid g\right) x_{i}\left(f, g^{\prime}\right),
$$

for every player $i$ and every graph $g \in G$. By definition,

$$
x_{i}(f, g)-x_{j}(f, g)=f_{i}(g)-f_{j}(g)+\delta \sum_{g^{\prime}} P\left(g^{\prime} \mid g\right)\left[x_{i}\left(f, g^{\prime}\right)-x_{j}\left(f, g^{\prime}\right)\right],
$$

and

$$
\begin{gathered}
x_{i}(f, g \backslash(i, j))-x_{j}(f, g \backslash(i, j))=f_{i}(g \backslash(i, j))-f_{j}(g \backslash(i, j))+ \\
+\delta \sum_{g^{\prime}} P\left(g^{\prime} \mid g \backslash(i, j)\right)\left[x_{i}\left(f, g^{\prime}\right)-x_{j}\left(f, g^{\prime}\right)\right] .
\end{gathered}
$$


From (5.1),

$$
\begin{gathered}
x_{i}(f, g)-x_{j}(f, g)=f_{i}(g)-f_{j}(g)+\delta \sum_{g_{1}:(i, j) \in g_{1}} P\left(g_{1} \mid g\right)\left[x_{i}\left(f, g_{1}\right)-x_{j}\left(f, g_{1}\right)\right] \\
+\delta \sum_{g_{2}:(i, j) \notin g_{2}} P\left(g_{2} \mid g\right)\left[x_{i}\left(f, g_{2}\right)-x_{j}\left(f, g_{2}\right)\right] .
\end{gathered}
$$

For every $g_{1}$ there is exactly one $g_{2}$ such that $g_{2}=g_{1} \backslash(i, j)$. By expected fairness of $f$ we have that for every such $g_{1}$ and $g_{2}$,

$$
x_{i}\left(f, g_{1}\right)-x_{j}\left(f, g_{1}\right)=x_{i}\left(f, g_{2}\right)-x_{j}\left(f, g_{2}\right)
$$

and thus

$$
\begin{gathered}
x_{i}(f, g)-x_{j}(f, g)=f_{i}(g)-f_{j}(g)+ \\
+\delta \sum_{g^{\prime} \ni(i, j)}\left[P\left(g^{\prime} \mid g\right)+P\left(g^{\prime} \backslash(i, j) \mid g\right)\right]\left[x_{i}\left(f, g^{\prime}\right)-x_{j}\left(f, g^{\prime}\right)\right] .
\end{gathered}
$$

Applying the same procedure to (5.2) we get that

$$
\begin{gathered}
x_{i}(f, g \backslash(i, j))-x_{j}(f, g \backslash(i, j))=f_{i}(g \backslash(i, j))-f_{j}(g \backslash(i, j))+ \\
+\delta \sum_{g^{\prime} \ni(i, j)}\left[P\left(g^{\prime} \mid g \backslash(i, j)\right)+P\left(g^{\prime} \backslash(i, j) \mid g \backslash(i, j)\right)\right]\left[x_{i}\left(f, g^{\prime}\right)-x_{j}\left(f, g^{\prime}\right)\right] .
\end{gathered}
$$

If $P$ satisfies the sum-property, then,

$$
P\left(g^{\prime} \mid g\right)+P\left(g^{\prime} \backslash(i, j) \mid g\right)=P\left(g^{\prime} \mid g \backslash(i, j)\right)+P\left(g^{\prime} \backslash(i, j) \mid g \backslash(i, j)\right)
$$

for every $g^{\prime}$ with $(i, j) \in g^{\prime}$.

Since $f$ is expected fair, we have

$$
x_{i}(f, g)-x_{j}(f, g)=x_{i}(f, g \backslash(i, j))-x_{j}(f, g \backslash(i, j)),
$$

and hence,

$$
f_{i}(g)-f_{j}(g)=f_{i}(g \backslash(i, j))-f_{j}(g \backslash(i, j)) .
$$

Therefore, if $P$ satisfies the sum-property, then an allocation rule is expected fair if and only if it is fair. But there is only one allocation rule which is component efficient and fair, namely the Myerson value. Hence, the unique allocation rule which is component efficient and expected fair is equal to the Myerson value, if $P$ satisfies the sum property. This completes the proof of Theorem 5.4. 


\section{An Example}

The following example shows that if the transition probability matrix $P$ does not satisfy the sum-property, then the component efficient and expected fair allocation rule may be different from the Myerson value for all $\delta \in(0,1)$.

Example 1. Let $N=\{1,2,3\}$. The set of all possible graphs on $N$ is given by

$$
G=\left\{\begin{array}{l}
g_{1}=g^{N}=\{(1,2),(1,3),(2,3)\} \\
g_{2}=\{(1,3),(2,3)\} \\
g_{3}=\{(1,2),(2,3)\} \\
g_{4}=\{(1,2),(1,3)\} \\
g_{5}=\{(1,3)\} \\
g_{6}=\{(2,3)\} \\
g_{7}=\{(1,2)\} \\
g_{8}=\{\{\varnothing\}\}
\end{array}\right.
$$

Let $w$ be equal to

$$
w(S, g)= \begin{cases}0, & \text { if }|S|=1 \\ 1, & \text { if }|S|=2 \\ 3, & \text { otherwise }\end{cases}
$$

Let the transition probability matrix $P$ be as follows.

$$
P\left(g_{h} \mid g_{k}\right)= \begin{cases}1, & \text { if }[h=k \neq 2] \text { or }[h=1 \text { and } k=2] \\ 0, & \text { otherwise. }\end{cases}
$$

Hence, the only non-trivial transition that occurs is from $g_{2}$ to $g_{1}$, with probability 1 .

Notice that

$$
P\left(g_{1} \mid g_{2}\right)+P\left(g_{4} \mid g_{2}\right) \neq P\left(g_{1} \mid g_{5}\right)+P\left(g_{4} \mid g_{5}\right),
$$

with $g_{1}=g_{4} \cup(2,3)$ and $g_{2}=g_{5} \cup(2,3)$. Thus, the matrix $P$ above does not satisfy the sumproperty. We will show that there is a unique component efficient and expected fair allocation rule which is different from Myerson value. The reader may check that the unique component efficient and expected fair allocation rule satisfies

$$
\begin{aligned}
& f_{1}\left(g_{2}\right)=\frac{5-6 \delta}{6(1-\delta)}=\frac{5}{6}-\frac{\delta}{6(1-\delta)}, \\
& f_{2}\left(g_{2}\right)=\frac{5-6 \delta}{6(1-\delta)}=\frac{5}{6}-\frac{\delta}{6(1-\delta)}, \\
& f_{3}\left(g_{2}\right)=\frac{4-3 \delta}{3(1-\delta)}=\frac{4}{3}+\frac{\delta}{3(1-\delta)},
\end{aligned}
$$

whereas the Myerson value yields $m\left(g_{2}\right)=\left(\frac{5}{6}, \frac{5}{6}, \frac{4}{3}\right)$. Hence, players 1 and 2 get less in $f$ than in the Myerson value, whereas for player 3 it is the opposite. 
The intuition is the following. Assume that we start from graph $g_{2}$ and consider the payoffs assigned to players 1 and 3 . Notice that in the case of fairness, we only compare the stage payoffs in $g_{2}$ with the stage payoffs players get in $g_{2} \backslash(1,3)$, which is the graph $g_{6}$. If link $(1,3)$ is not present, in other words, if the actual structure is given by graph $g_{6}$ player 3 will get a payoff of $\frac{1}{2}$ and player 1 will get 0 . By imposing expected fairness, we take into account the following. If link $(1,3)$ is not present, the difference in payoffs is $\frac{1}{2}$ from now on, discounted by $\delta$. On the other hand, if $(1,3)$ is present, players will move in the next stage to graph $g_{1}$, and stay there forever. In $g_{1}$ the difference in payoffs is 0 . Thus, fairness requires that

$$
m_{3}\left(g_{2}\right)-m_{1}\left(g_{2}\right)=\frac{1}{2}
$$

whereas expected fairness requires

$$
f_{3}\left(g_{2}\right)-f_{1}\left(g_{2}\right)+\delta \times 0+\delta^{2} \times 0+\delta^{3} \times 0 \ldots=\frac{1}{2}+\delta \times \frac{1}{2}+\delta^{2} \times \frac{1}{2}+\delta^{3} \times \frac{1}{2} \ldots
$$

which means

$$
f_{3}\left(g_{2}\right)-f_{1}\left(g_{2}\right)=\frac{1}{2} \frac{1}{1-\delta}
$$

We therefore observe that the difference in payoffs when we consider expected fairness goes to the difference in the Myerson value when $\delta$ tends to zero, and it goes to infinity when $\delta$ goes to 1 .

\section{Appendix}

Proof of Theorem 2.5. By Theorem 2.3, there is a unique allocation rule which is component efficient and fair. It thus suffices to show that the allocation rule $y(g)=\varphi^{M}\left(N, U_{g}\right)$ for all $g$ is indeed component efficient and fair. In order to do so, we need the following axiomatic characterization for $\varphi^{M}$ provided by Myerson [10].

Definition 7.1. An allocation rule $\varphi$ is called efficient if for every game in PFF $\langle N, v\rangle$

$$
\sum_{i \in N} \varphi_{i}(N, v)=v(N,\{N\})
$$

Definition 7.2. An allocation rule $\varphi$ is called additive if for every two games $\left\langle N, v_{1}\right\rangle$ and $\left\langle N, v_{2}\right\rangle$

$$
\varphi\left(N, v_{1}+v_{2}\right)=\varphi\left(N, v_{1}\right)+\varphi\left(N, v_{2}\right) .
$$

Let $\pi$ be a permutation on the set of players. Let $\pi(S)=\{\pi(i): i \in S\}$ and $\pi(P)=$ $\left\{\pi\left(S_{1}\right), \ldots, \pi\left(S_{r}\right)\right\}$, where $\left\{S_{1}, \ldots, S_{r}\right\}=P$. For any coalitional value function $v$ and permutation $\pi$ we define the coalitional value function $\pi \circ v$ as $(\pi \circ v)(\pi(S), \pi(P))=v(S, P)$, for any pair $(S, P) \in E(N)$. 
Definition 7.3. An allocation rule $\varphi$ is symmetric if for any permutation $\pi$, any coalitional value function $v$ and any player $i \in N$,

$$
\varphi_{\pi(i)}(N, \pi \circ v)=\varphi_{i}(N, v) .
$$

For any two partitions $P, P^{\prime}$ on $N$, we define the partition $P \wedge P^{\prime}$ on $N$ as

$$
P \wedge P^{\prime}=\left\{S \cap S^{\prime} \mid S \in P, S^{\prime} \in P^{\prime} \text { and } S \cap S^{\prime} \neq \varnothing\right\} .
$$

For $\langle N, v\rangle$ given, a coalition $S \subseteq N$ is a carrier of $\langle N, v\rangle$ if for all embedded coalitions $(T, P)$ $v(T, P)=v(T \cap S, P \wedge\{S, N \backslash S\})$.

Definition 7.4. An allocation rule $\varphi$ satisfies the carrier property if for every $\langle N, v\rangle$ and every coalition $S$, if $S$ is a carrier of $\langle N, v\rangle$, then

$$
\sum_{i \in S} \varphi_{i}(N, v)=v(N,\{N\})
$$

Proposition 7.5. (Myerson [10]) There is a unique allocation rule which is efficient, additive, symmetric and satisfies the carrier property, namely $\varphi^{M}$.

Claim 1. The allocation rule $\varphi^{M}\left(N, U_{g}\right)$ is component efficient.

Proof of Claim 1. Following Feldman [5], we can define for every connected component $T \in N \mid g$ a game in $\operatorname{PFF}\left\langle N, u_{g}^{T}\right\rangle$ as follows. For every $(S, P) \in E(N)$

$$
u_{g}^{T}(S, P)=\sum_{R \in(S \cap T) \mid g} w(R, g \mid P) .
$$

We show now that $T$ is a carrier in the game $\left\langle N, u_{g}^{T}\right\rangle$. In order to do so, we need to prove that $g|P=g|(P \wedge\{T, N \backslash T\})$. Choose any $R \in P$. Note that for every pair of players $i$ and $j$ in $R$, with $i \in T$ and $j \notin T$ the link $(i, j) \notin g$. Therefore, $(i, j) \notin g \mid R$, for $g \mid R$ is always a subgraph of $g$. It thus follows that

$$
g|R=[g \mid(R \cap T)] \cup[g \mid(R \cap(N \backslash T))]=g| R^{\prime} \cup g \mid R^{\prime \prime},
$$

where $R^{\prime} \cup R^{\prime \prime}=R$ and $R^{\prime}=R \cap T, R^{\prime \prime}=R \cap(N \backslash T)$. Notice $R^{\prime}$ and $R^{\prime \prime} \in P \wedge\{T, N \backslash T\}$ whenever they are non empty.

But this means that

$$
\bigcup_{R \in P} g\left|R=\bigcup_{R \in P}\left(g\left|R^{\prime} \cup g\right| R^{\prime \prime}\right)=\bigcup_{\tilde{R} \in P \wedge\{T, N \backslash T\}} g\right| \tilde{R},
$$

and thus $g|P=g|(P \wedge\{T, N \backslash T\})$. Therefore, for any $T \in N \mid g$

$$
u_{g}^{T}(S, P)=u_{g}^{T}(S, P \wedge\{T, N \backslash T\})=u_{g}^{T}(S \cap T, P \wedge\{T, N \backslash T\}) .
$$


In other words, $T$ is a carrier of $\left\langle N, u_{g}^{T}\right\rangle$. By the carrier property of $\varphi^{M}$, for any $S \in N \mid g$

$$
\sum_{i \in S} \varphi_{i}^{M}\left(N, u_{g}^{T}\right)=\left\{\begin{array}{cc}
u_{g}^{T}(N,\{N\}), & \text { if } S=T \\
0, & \text { otherwise. }
\end{array}\right.
$$

On the other hand, note that $N \mid g$ denotes a partition on $N$. Furthermore, any two players which are not connected in $g$ will not be connected either in $g \mid S$, for any $S$. Thus,

$$
U_{g}(S, P)=\sum_{T \in N \mid g} u_{g}^{T}(S, P)
$$

By linearity of $\varphi^{M}$, for any $S \in N \mid g$

$$
\sum_{i \in S} \varphi_{i}^{M}\left(N, U_{g}\right)=\sum_{T \in N \mid g} \sum_{i \in S} \varphi_{i}^{M}\left(N, u_{g}^{T}\right)=u_{g}^{S}(N,\{N\}) .
$$

By definition, $u_{g}^{S}(N,\{N\})=\sum_{R \in(S \cap N) \mid g} w(R, g \mid\{N\})=w(S, g)$, for any $S \in N \mid g$. Thus, the allocation rule $\varphi^{M}$ is component efficient, which completes the proof of Claim 1.

Claim 2. The allocation rule $\varphi^{M}\left(N, U_{g}\right)$ is fair.

Proof of Claim 2. Fix a graph $g$. For every $(i, j) \in g$ define the game in $\operatorname{PFF}\left\langle N, V_{g,(i, j)}\right\rangle$, where $V_{g,(i, j)}=U_{g}-U_{g \backslash(i, j)}$. We will show that $\varphi_{i}^{M}\left(N, V_{g,(i, j)}\right)=\varphi_{j}^{M}\left(N, V_{g,(i, j)}\right)$, which by linearity of $\varphi^{M}$ implies that $\varphi^{M}\left(N, U_{g}\right)$ is fair.

Note that $V_{g,(i, j)}(S, P)=0$ for any partition $(S, P) \in E(N)$ such that players $i$ and $j$ are in different sets in the partition $P$. Therefore, only the embedded coalitions $(S, P)$ such that players $i$ and $j$ belong to the same set in $P$, say $S_{i j} \in P$, have a non zero value. Thus,

$$
\begin{aligned}
& \varphi_{i}^{M}\left(N, V_{g,(i, j)}\right)-\varphi_{j}^{M}\left(N, V_{g,(i, j)}\right)=\sum_{\substack{(S, P) \in E(N) \\
(i, j) \subseteq S_{i j} \in P}}(-1)^{|P|-1}(|P|-1) ! \times \\
& \times\left(\sum_{\substack{\tilde{S} \in P \\
\tilde{S} \neq S \\
j \notin \tilde{S}}} \frac{1}{(|P|-1)(n-|\tilde{S}|)}-\sum_{\substack{\tilde{S} \in P \\
\tilde{S} \neq S \\
i \notin \tilde{S}}} \frac{1}{(|P|-1)(n-|\tilde{S}|)}\right) V_{g,(i, j)}(S, P) .
\end{aligned}
$$

Notice that if players $i$ and $j$ belong to the same coalition $S_{i j} \in P$, the sets

$$
\{\tilde{S}: \tilde{S} \in P, \tilde{S} \neq S \text { and } i \notin \tilde{S}\}
$$

and

$$
\{\tilde{S}: \tilde{S} \in P, \tilde{S} \neq S \text { and } j \notin \tilde{S}\}
$$


are both equal to the set

$$
\left\{\tilde{S}: \tilde{S} \in P, \tilde{S} \neq S \text { and } \tilde{S} \neq S_{i j}\right\}
$$

This means that

$$
\sum_{\substack{\tilde{S} \in P \\ \tilde{S} \neq S \\ j \notin \tilde{S}}} \frac{1}{(|P|-1)(n-|\tilde{S}|)}-\sum_{\substack{\tilde{S} \in P \\ \tilde{S} \neq S \\ i \notin \tilde{S}}} \frac{1}{(|P|-1)(n-|\tilde{S}|)}=0
$$

for any $(S, P) \in E(N)$, with players $i$ and $j$ belonging to the same coalition $S_{i j} \in P$. From (7.1) and (7.2) we obtain that $\varphi_{i}^{M}\left(N, V_{g,(i, j)}\right)-\varphi_{j}^{M}\left(N, V_{g,(i, j)}\right)=0$. This completes the proof of Claim 2.

By Claims 1 and 2 we have shown that the allocation rule $\varphi^{M}\left(N, U_{g}\right)$ is component efficient and fair. This completes the proof of Theorem 2.5.

\section{References}

[1] Bala, V., \& S. Goyal (2000), A Strategic Analysis of Network Reliability. Rev. Econ. Design 5(3): 205-228.

[2] Bolger, E. M. (1986), Power Indices for Multicandidate Voting Games. Int. J. Game Theory 15 (3): 175-186.

[3] Calvo, E., Lasaga, J. and A. van den Nouweland (1999), Values of Games with Probabilistic Graphs. Math. Soc. Sci. 37: 79-95.

[4] Calvo-Amengol, A. and M. O. Jackson (2002), Social Networks in Determining Employment and Wages: Patterns, Dynamics and Inequality. Mimeo.

[5] Feldman, B. E. (1996), Bargaining, Coalition Formation and Value. Ph.D. Dissertation. State University of New York at Stony Brook.

[6] Jackson, M. O. \& A. Watts (2002), The Evolution of Social and Economic Networks. J. Econ. Theory 106 (2): 265-295.

[7] Jackson, M. O. \& A. Wolinsky (1996), A Strategic Model of Social and Economic Networks. J. Econ. Theory 71: 44-74.

[8] Lucas, W. F. \& R. M. Thrall (1963), n-Person Games in Partition Function Form. Naval Research Logistics Quarterly 10: 281-298.

[9] Myerson, R. B. (1977a), Graphs and Cooperation in Games. Mathematics of Operations Research, 2: 225-229.

[10] Myerson, R. B. (1977b), Values of Games in Partition Function Form. Int. J. Game Theory 6 (1): 23-31. 